\title{
LA DOCENCIA EN LA UNIVERSIDAD Y EL COMPROMISO SOCIAL Y EDUCATIVO
}

\author{
Sara Ibarrola-García \\ Raquel Artuch Garde \\ Universidad de Navarra
}

\begin{abstract}
RESUMEN: La Educación Superior contribuye a la formación de competencias para el mundo de hoy y de mañana, pero al mismo tiempo a la formación de futuros profesionales responsables socialmente, con principios éticos, comprometidos con la construcción de la paz, la defensa de los derechos humanos y los valores de la democracia (UNESCO, 2009). En este trabajo se destaca el Aprendizaje-Servicio como metodología que permite integrar el aprendizaje académico con el servicio solidario a la comunidad con el fin de mejorarla. En primer lugar, se analiza la difusión actual del aprendizajeservicio en el contexto universitario español. Se ahonda en el impacto positivo que tiene en los alumnos describiendo las evidencias halladas en la bibliografía. En segundo lugar, se describe y se evalúa una experiencia llevada a cabo en el Grado de Educación de la Universidad de Navarra durante los cursos 2013-2014 y 2014-2015. Se recogen las percepciones sobre los resultados de aprendizaje alcanzados por los alumnos implicados. Estos resultados sugieren que la experiencia ha favorecido la adquisición de los contenidos de la asignatura al mismo tiempo que algunas competencias participativas que se detallan, destacando especialmente el componente social.
\end{abstract}

PALABRAS CLAVE: Aprendizaje-servicio, competencias del docente, metodología, innovación pedagógica, formación inicial del docente.

\section{TEACHING AT THE UNIVERSITY AND SOCIAL AND EDUCATIONAL IMPROVEMENT}

ABSTRACT: Higher Education contributes not only to the formation of skills for the world of today and tomorrow, but also to the training of socially responsible future professionals with ethical principles, who are committed to working for peace, human rights and the values of democracy (UNESCO, 2009). This work emphasizes service-learning as a methodology to integrate 
academic learning, along with community service solidarity as a method of improving it. Firstly, the spread of service-learning in the context of a Spanish university is analyzed. It delves into the positive impact it has on students. The evidence found in the literature is described. Secondly, a service-learning experience carried out in the Degree of Education from the University of Navarra is described and evaluated. Perceptions of the learning outcomes achieved by the students involved are collected. These results suggest that the experience has led to a high level of acquisition of the contents of the subject and its related skills, with particular emphasis on the social component.

KEYWORDS: Service-learning, teacher competences, methodology, educative innovation, preservice teacher education.

Recibido: 29/04/2015

Aceptado: 24/07/2015

Correspondencia: Sara Ibarrola García, Departamento de Aprendizaje y Curriculum, Facultad de Educación y Psicología, Campus Universidad de Navarra, Edificio de Bibliotecas, 31009 Pamplona. Email: sigarcia@unav.es.

\section{INTRODUCCIÓN}

En la sociedad del conocimiento y en el marco actual del Espacio Europeo de Educación Superior (EEES), el enfoque de las competencias ha traído consigo una serie de cambios que afectan al proceso de enseñanza-aprendizaje y muy particularmente a la metodología empleada, clave en los nuevos modelos de formación del profesorado.

En la Declaración de Bolonia de 1999, ante el reto de construir el área europea de educación superior, se concretaron una serie de objetivos con el fin de proporcionar a los universitarios las competencias necesarias para el desarrollo y el fortalecimiento de sus dimensiones intelectual, cultural, social, científica y tecnológica (párrafo 1).

Para llevarlo a cabo se han adoptado una serie de medidas legislativas. Entre ellas, la Ley Orgánica de Universidades de 2007 señala la necesidad de una mayor implicación de las universidades en la mejora de la formación de sus graduados para que sean capaces de adaptarse tanto a las demandas sociales como a las demandas del sistema científico y tecnológico. Y el Real Decreto 1393/2007 por el que se establece la ordenación de las enseñanzas universitarias oficiales, determina que los planes de estudios conducentes a la obtención de un título deberán tener en el centro de sus objetivos la adquisición de competencias por parte de los estudiantes, ampliando el tradicional enfoque basado en contenidos (preámbulo, párrafo 8).

El enfoque por competencias precisa poner en marcha en las aulas universitarias nuevas orientaciones y estrategias metodológicas. Ya no se espera una transmisión unidireccional de los contenidos sino que, a la hora de programarlos y seleccionarlos, el profesor universitario necesita tener en cuenta que el alumno llegará a ser competente cuando sea capaz de transferir dichos conocimientos a diferentes contextos. 
En esta línea, se favorecerá el aprendizaje por competencias si el profesor universitario diseña escenarios de trabajo que se aproximen a la práctica o que al menos necesiten contar con su referente. De acuerdo con Cornish y Perkins (2012) plantear en el aula pseudoprácticas o situaciones hipotéticas supone una manera más fácil de pensar sobre la teoría, lo que requiere la combinación de diversas metodologías docentes junto a la lección magistral como por ejemplo el estudio de casos, el trabajo cooperativo, los seminarios o el aprendizaje basado en problemas.

Como afirma Pérez-Gómez (2010), integrar en la docencia universitaria los referentes del contexto real de la práctica facilitarán que el conocimiento teórico adquiera significación, relevancia y durabilidad. Se pasa así, siguiendo a Bernstein (1993), de un contexto donde el conocimiento sólo se transmite, a un contexto donde se utiliza como herramienta para el análisis, la comprensión y la toma de decisiones para la práctica.

En esta línea se apuesta por la introducción de metodologías innovadoras que en la medida de lo posible planteen situaciones cercanas al contexto profesional en el que se desenvolverán los alumnos en un futuro, haciéndoles partícipes y responsables de una actividad compleja que exige aprendizaje significativo. Ello, sin perder de vista que la Educación Superior debe no sólo proporcionar competencias sólidas para el mundo de hoy y de mañana, sino contribuir al mismo tiempo a la información de una ciudadanía dotada de principios éticos, comprometida con la construcción de la paz, defensa de los derechos humanos y valores de la democracia (UNESCO, 2009). Ambas dimensiones, cualificación y responsabilidad social, forman un binomio inseparable.

Por esta razón, entre otras metodologías innovadoras destacamos el AprendizajeServicio como "propuesta educativa que combina procesos de aprendizaje y de servicio a la comunidad en un único proyecto bien articulado en el que los participantes aprenden a la vez que trabajan en necesidades reales del entorno con la finalidad de mejorarlo" (Puig, Batlle, Bosch y Palos, 2007: 20).

\section{El APRENDIZAJE-SERVICIO EN LA UNIVERSIDAD: CONCEPTO Y BENEFICIOS}

El aprendizaje-servicio, o su traducción anglosajona service-learning, nació en los Estados Unidos a mediados del s. XX. Según Halstead (1998) en los años 90 ya estaba consolidada en ese país como herramienta ampliamente utilizada en las instituciones educativas a todos los niveles. Incluso ya había dado el salto a otros países. Durante este proceso ha sido necesario esclarecer los elementos configuradores propios del aprendizaje-servicio para no confundirlo, como en ocasiones ha ocurrido, con otros términos afines como voluntariado, acciones voluntarias esporádicas o trabajo de campo. Estos son los siguientes (Martínez-Odría, 2005): a) conexión con los objetivos curriculares de aprendizaje, b) atención a una necesidad real que surge desde la comunidad, c) ejecución de un proyecto servicio, d) protagonismo de la voz del alumno, y e) reflexión.

Se trata, en definitiva, de un método o enfoque educativo y de intervención social que pone en interacción el aprendizaje académico del alumnado con el servicio solidario que éste realiza, para contribuir a la calidad educativa con un sentido de responsabilidad social. 
Durante la última década en España ha tenido lugar una gran difusión del aprendizaje-servicio en las instituciones educativas de educación superior (Batlle, 2014). Consecuentemente su presencia se ha acelerado en los últimos años. Muestra de ello ha sido el estudio de las bases teóricas y conceptuales de esta metodología (MartínezOdría, 2005), la proliferación de investigaciones que se están desarrollando en distintas universidad españolas (Campo, 2010; Cerrillo, García y López, 2013; Folgueiras, Luna y Puig, 2013; Gil, Chiva y Martí, 2014, Rodríguez, 2014), la presencia de este término en los textos oficiales, la constitución de equipos de formadores-asesores comprometidos liderados desde 2008 por la profesora Roser Batlle, y la construcción de la Red Española de Aprendizaje-Servicio, que engloba la red ApS(U), red específica del profesorado universitario.

Este considerable aumento de experiencias puede deberse en parte a su virtualidad educativa. Diferentes estudios (Billing y Waterman, 2014; Bringe y Hatcher, 2009; Hérbet y Hauf, 2015; Howard, 2003) han puesto de manifiesto el impacto positivo que el aprendizaje-servicio tiene en el profesorado, la institución educativa o la propia comunidad local y en los alumnos. Precisamente Furco (1999) indica que las ventajas del aprendizaje-servicio revierten especialmente en el aprendizaje académico de los alumnos, en el desarrollo de habilidades y actitudes que favorecen un servicio de calidad a la comunidad y en la comprensión de la problemática social en la que se inserta el proyecto.

Martínez-Odría (2006) asimismo indica cómo el aprendizaje-servicio contribuye a desarrollar las distintas competencias genéricas definidas:

Tabla 1. Conjunto de competencias genéricas que contribuye a desarrollar el service-learning (Martínez-Odría, 2006)

\begin{tabular}{lcc}
\hline \multicolumn{1}{c}{$\begin{array}{c}\text { Competencias } \\
\text { instrumentales }\end{array}$} & \multicolumn{1}{c}{$\begin{array}{c}\text { Competencias } \\
\text { interpersonales }\end{array}$} & $\begin{array}{c}\text { Competencias } \\
\text { sistémicas }\end{array}$ \\
\hline - capacidad de análisis y & - capacidad crítica y & - capacidad de aplicar los \\
síntesis; & autocrítica; & conocimientos en la práctica; \\
- capacidad de organizar y & - trabajo en equipo; & - habilidades de investigación; \\
planificar; & - habilidades interpersonales; & - capacidad de aprender; \\
- conocimientos básicos de la & - capacidad de trabajar en un & - capacidad para adaptarse a \\
profesión; & equipo interdisciplinar; & nuevas situaciones; \\
- comunicación oral y escrita & - capacidad para comunicarse & - capacidad para generar \\
en la propia lengua; & con expertos de otras áreas; & nuevas ideas (creatividad); \\
- habilidades de gestión de la & - apreciación de la diversidad & - liderazgo; \\
información (habilidad para & y la multiculturalidad; & - conocimiento de culturas y \\
buscar y analizar información & - habilidad de trabajar en un & costumbres de otros países; \\
proveniente de fuentes & contexto internacional; & - habilidad para trabajar de \\
diversas); & - compromiso ético & forma autónoma; \\
- resolución de conflictos; & & - diseño y gestión de \\
- toma de decisiones & & proyectos; \\
& & - iniciativa y espíritu \\
& & emprendedor; \\
& & - preocupación por la calidad \\
& & y motivación de logro \\
\hline
\end{tabular}


Sobre este marco teórico y en esta línea de actuación puede ser muy útil evaluar las experiencias que se lleven a cabo para conocer sus resultados. Evaluar los diferentes pasos que se vayan siguiendo y la satisfacción o las percepciones que va generando, dar visibilidad a los aspectos positivos y a sus ventajas puede suponer una progresiva sensibilización e incluso un reclamo para la incorporación de metodologías innovadoras como el aprendizaje-servicio. En este sentido, este artículo tiene como pretensión presentar una experiencia de aprendizaje-servicio realizada con alumnos universitarios donde los resultados y el procedimiento, de carácter local y tomado con cautela, sirvan como ejemplo para trabajos similares en otras universidades.

\section{UnA EXPERIENCIA DE APRENDIZAJE-SERVICIO en la ASIGNATURA “APrendizAJE DE LENGUAS Y ATENCIÓN A LA DIVERSIDAD LINGÜÍSTICA"}

\section{Descripción de la experiencia}

La experiencia piloto se desarrolló durante el primer cuatrimestre de los cursos académicos 2013-2014 y 2014-2015, por primera y segunda vez respectivamente, en la asignatura "Aprendizaje de lenguas y atención a la diversidad lingüística", impartida en los Grados de Educación Primaria y Educación Infantil. Esta asignatura persigue que el alumno conozca y adquiera recursos para planificar los procesos de enseñanza-aprendizaje de lenguas y diversidad lingüística.

\section{Objetivos}

Los objetivos planteados fueron los siguientes:

1. Conocer si los alumnos de la asignatura perciben que el trabajo realizado ha facilitado la adquisición de los contenidos de la asignatura.

2. Conocer la opinión de los alumnos de la asignatura en cuanto a su grado de adquisición y desarrollo de habilidades y actitudes participativas.

3. Conocer si los alumnos de la asignatura consideran que el trabajo realizado ha influido en su modo de percibir la realidad social.

4. Conocer el tipo de relación existente entre los conocimientos adquiridos y las competencias participativas (habilidades y actitudes) desarrolladas.

5. Conocer si existen diferencias significativas entre años académicos (20132014/2014-2015) y curso escolar $\left(3^{\circ}\right.$ y $\left.5^{\circ}\right)$ en la percepción de conocimientos adquiridos, el desarrollo de habilidades y actitudes participativas.

\section{Hipótesis}

H1. El trabajo de aprendizaje-servicio realizado facilita la adquisición de los contenidos de la asignatura al planificar la actividad, al ponerla en práctica y reflexionar sobre ella.

H2. El grado de adquisición y desarrollo de habilidades y actitudes participativas será positivo. 
H3. El trabajo realizado en la asignatura permitirá a los alumnos conocer más la realidad social.

H4. Existe relación entre los conocimientos adquiridos y las habilidades desarrolladas.

H5. Consideramos que existirán diferencias significativas en el nivel de desarrollo y adquisición de competencias en función del curso académico $\left(3^{\circ} \mathrm{o} 5^{\circ}\right)$.

\section{Método}

\section{Participantes}

La muestra la conformaron 62 alumnos que cursaron la asignatura de "Aprendizaje de lenguas y atención a la diversidad lingüística" durante los cursos 2013-2014 $(n=31)$ y 2014-2015 ( $n=31)$. El grupo de alumnos de la asignatura cursaba $3^{\circ}$ de Educación Infantil y Educación Primaria y $5^{\circ}$ del Doble Grado de Pedagogía y Educación Infantil.

\section{Instrumentos}

Se utilizó como instrumento de evaluación un "Cuestionario sobre la experiencia de apoyo lingüístico en la Fundación Core" realizado al efecto. Para la elaboración del cuestionario se tuvieron en cuenta dos aspectos. Por un lado, las tres dimensiones en las que los alumnos universitarios pueden desarrollar aprendizajes a través de su implicación en una experiencia de aprendizaje-servicio (Furco, 1999): 1) la dimensión académica, 2) la dimensión de desarrollo profesional, y 3) la dimensión social.

Por otra parte como resalta Lucas (2012), para desarrollar todo su potencial pedagógico es indispensable que se lleve a cabo a través de una buena preparación y planificación previa a la acción, así como la reflexión crítica posterior y la evaluación a lo largo del proceso de la adquisición autónoma de los conocimientos necesarios por parte del alumnado. Por tanto se distinguen tres momentos cruciales en un proyecto de aprendizaje-servicio: la planificación, la acción y la reflexión.

De manera que el cuestionario se estructura en tres bloques (componente académico, componente de habilidades y actitudes y componente social respectivamente) y su contenido contempla y diferencia ítems referidos a aprendizajes que los alumnos realizaron en tres momentos diferentes: durante la preparación y planificación de la acción, durante la acción y después de la acción.

\section{Procedimiento}

La experiencia de aprendizaje-servicio desarrollada consistió en realizar un proyecto de "apoyo lingüístico a alumnos inmigrantes". Para ello se contó con la colaboración de la Fundación Core de Pamplona, una fundación de interés social y sin ánimo de lucro que pretende apoyar a las personas inmigrantes en su proceso de integración social, educativa y laboral a través del desarrollo de diversas actividades y programas formativos. La Fundación Core facilitó el acceso a niños inmigrantes que 
cursan Educación Infantil y Educación Primaria. En primer lugar, se formaron grupos de 4-5 universitarios y se pidió a los alumnos que prepararan actividades en torno a la temática del otoño a través de las cuales trabajar aspectos lingüísticos. Cada grupo debía preparar dos sesiones de 90 minutos de duración. En segundo lugar, cada grupo, acompañado por una profesora de la universidad, acudió a la Fundación durante los meses de septiembre a diciembre para ofrecer apoyo y poner en práctica las actividades programadas.

La profesora hizo un seguimiento del trabajo ofreciendo asesoramiento durante su diseño y también durante su realización in situ cuando los alumnos así lo requirieron. Cada día al finalizar la sesión los alumnos debían rellenar una ficha de registro en la que anotaban lo que habían realizado en la actividad en función de los objetivos previstos, así como las observaciones de cada uno de los niños. Para que cada grupo pudiera coordinarse con el grupo-clase y compartir la información entre todos los compañeros, se asignó a cada niño una carpeta en la que cada grupo incluía toda la información y lo entregaba al siguiente grupo responsable.

También al final de la asignatura los alumnos individualmente elaboraron una memoria donde realizaron una reflexión guiada sobre la actividad en su conjunto.

Finalmente, el último día de clase de la asignatura, se pidió a los alumnos que cumplimentaran el cuestionario a modo de autoinforme reflexivo a través del cual se quería conocer su opinión sobre el grado de adquisición y desarrollo de algunas competencias.

Con todo ello se intentó contemplar en la dinámica de trabajo aquellos aspectos que según Lucas (2012) favorecen la eficacia de las experiencias de aprendizaje-servicio, estos son: la planificación de las sesiones de trabajo con la antelación necesaria; el control y el apoyo de la profesora sobre cómo presentar y organizar las actividades, cómo dinamizar los trabajos en grupo o cómo evaluar su realización; la información compartida dentro del grupo, con la profesora y el resto de grupos; la clarificación de objetivos, tareas y el plan a seguir por cada grupo; y, la evaluación de la actuación del grupo en función de las propuestas y acciones realizadas.

Los alumnos, cuya participación en la cumplimentación del cuestionario fue voluntaria y a quienes se les garantizó la confidencialidad de los datos, lo completaron individualmente durante aproximadamente 30 minutos. Se mantuvo un ambiente de silencio para favorecer la reflexión.

\section{Análisis de datos}

Al tratarse de un estudio piloto sobre una experiencia de aprendizaje servicio con una muestra concreta y reducida, los resultados de los análisis realizados deben servir como orientación para futuras experiencias e investigaciones. En este sentido, en la medida de lo posible, se realizaron análisis descriptivos, de Asociación (Spearman) y análisis de diferencia de medias para pruebas no paramétricas ( U de Mann-Whitney).

Los análisis que se Ilevaron a cabo para cumplir con los objetivos propuestos y probar las hipótesis planteadas se realizaron utilizando el paquete estadístico SPSS versión 21.00 para Windows. 


\section{Resultados}

\section{Descriptivos}

Grado de adquisición de competencias relacionadas con la dimensión académica

En la siguiente tabla (ver Tabla 2) se observa el resultado de los ítems referidos al grado de acuerdo en cuando al nivel de desarrollo y adquisición de conocimientos por parte de los alumnos en relación a los objetivos, las actividades y las orientaciones metodológicas en el diseño, ejecución y reflexión de la experiencia realizada. En este sentido se puede comprobar cómo los alumnos señalan las mayores puntuaciones en los conocimientos adquiridos "tras la actividad". Entre estos, destacan que tras la misma se han dado cuenta de la importancia que tiene la metodología para orientar las actividades, de la importancia de ser coherente con el contenido y el objetivo lingüístico que se quería trabajar y la importancia de saber identificar en la práctica cómo plantear el desarrollo de las actividades.

Tabla 2. Conocimientos adquiridos

\begin{tabular}{lcc}
\hline & Media & DT \\
\hline $\begin{array}{l}\text { CA9. Tras la actividad. Darse cuenta de la importancia que tiene la ME- } \\
\text { TODOLOGÍA para orientar las actividades }\end{array}$ & 4.44 & 0.738 \\
$\begin{array}{l}\text { CA8. Tras la actividad. Darse cuenta de la importancia de ser COHEREN- } \\
\text { TE con el CONTENIDO y el OBJETIVO lingüístico }\end{array}$ & 4.29 & 0.637 \\
$\begin{array}{l}\text { CA7. Tras la actividad. Dar MÁS importancia que antes a SABER IDENTI- } \\
\text { FICAR en la práctica cómo plantear el DESARROLLO de las actividades }\end{array}$ & 4.11 & 0.791 \\
$\begin{array}{l}\text { CA3. Antes de la actividad. Tener en cuenta que las ACTIVIDADES estu- } \\
\text { vieran ORIENTADAS al LOGRO DEL OBJETIVO/S }\end{array}$ & 4.11 & 0.791 \\
$\begin{array}{l}\text { CA5. Durante la actividad. Tener presente el OBJETIVO/S que se } \\
\text { perseguía/n }\end{array}$ & 3.89 & 0.889 \\
$\begin{array}{l}\text { CA4. Antes de la actividad. Tener en cuenta las ORIENTACIONES METO- } \\
\text { DOLÓGICAS estudiadas en la asignatura }\end{array}$ & 3.87 & 0.839 \\
$\begin{array}{l}\text { CA6. Durante la actividad. Las orientaciones metodológicas estudiadas } \\
\text { me han ayudado a proceder mejor }\end{array}$ & 3.82 & 1.017 \\
\hline
\end{tabular}

En cuanto a si los alumnos se plantearon o no objetivos relacionados con alguna de las cuatro habilidades lingüísticas al planificar la actividad, el $87.1 \%$ respondieron que sí, mientras que el $12.9 \%$ restante señalaron que no lo hicieron.

Grado de adquisición de competencias relacionadas con la dimensión de desarrollo profesional

En relación a las competencias participativas desarrolladas, podemos destacar que los alumnos señalan fundamentalmente que la experiencia les ha ayudado a desarrollar aquellas relacionadas con la toma de decisiones, el trabajo en equipo y la iniciativa y proactividad. En menor medida han desarrollado competencias relacionadas con el liderazgo o la laboriosidad (ver Tabla 3). También se pueden 
observar en la tabla 4 los resultados de los ítems referidos a acciones que ponen en juego algunas habilidades profesionales durante la planificación, la puesta en práctica y a posteriori de la actividad realizada.

Tabla 3. Competencias participativas desarrolladas

\begin{tabular}{lccc}
\hline Habilidades y actitudes & & N & porcentaje \\
\hline \multirow{2}{*}{ Toma de decisiones } & SI & 58 & $93.5 \%$ \\
\hline \multirow{2}{*}{ Trabajo en equipo } & NO & 4 & $6.5 \%$ \\
\hline \multirow{2}{*}{ Iniciativa y proactividad } & $\mathrm{SI}$ & 57 & $91.9 \%$ \\
& $\mathrm{NO}$ & 5 & $8.1 \%$ \\
\hline \multirow{2}{*}{ Capacidad de análisis } & $\mathrm{SI}$ & 56 & $90.3 \%$ \\
& $\mathrm{NO}$ & 6 & $9.7 \%$ \\
\hline \multirow{2}{*}{ Capacidad de reflexión } & $\mathrm{SI}$ & 54 & $88.5 \%$ \\
& $\mathrm{NO}$ & 7 & $11.5 \%$ \\
\multirow{2}{*}{ Laboriosidad } & $\mathrm{SI}$ & 45 & $76.3 \%$ \\
\multirow{2}{*}{ Liderazgo } & $\mathrm{NO}$ & 14 & $23.7 \%$ \\
\hline
\end{tabular}
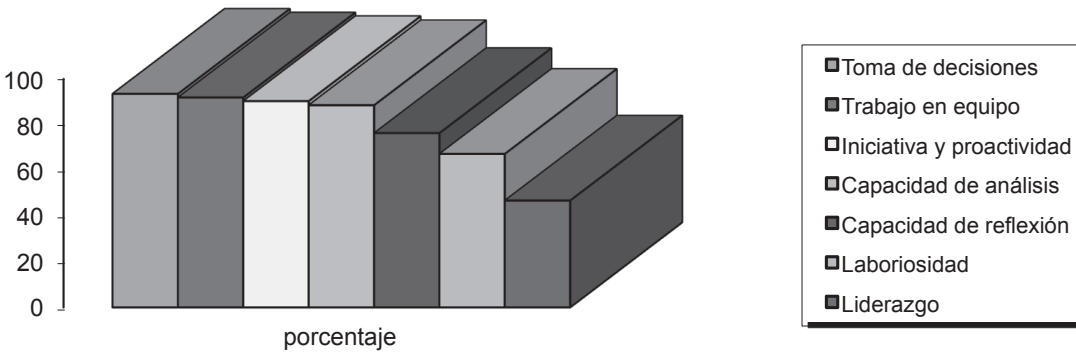

Gráfico 1. Porcentaje de competencias participativas desarrolladas

En cuanto a las competencias participativas desarrolladas, de nuevo se observa que la mayor puntuación se obtiene "tras la actividad", donde los alumnos señalan que ésta les ha ayudado a percibir la influencia que tiene la coordinación y organización con los compañeros. Además, "durante la actividad", señalan que se han enriquecido con la experiencia de sus compañeros y han sabido responder con flexibilidad a las necesidad es que se han presentado durante las sesiones. En el lado opuesto, los alumnos señalan que en menor medida han utilizado la información de las carpetas asignadas a cada niño para obtener información valiosa y tratar de planificar objetivos de trabajo claros. 
Tabla 4. Competencias participativas desarrolladas

\begin{tabular}{|c|c|c|}
\hline & Media & DT \\
\hline $\begin{array}{l}\text { Tras la actividad. Percibir la influencia que tiene la coordinación y } \\
\text { organización con mis compañeros en la práctica educativa }\end{array}$ & 4,38 & 0,687 \\
\hline Durante la actividad. Enriquecerse con la experiencia de los compañeros & 4,35 & 0,726 \\
\hline $\begin{array}{l}\text { Durante la actividad. Responder con flexibilidad a las necesidades que } \\
\text { se han presentado durante las sesiones }\end{array}$ & 4,34 & 0,571 \\
\hline $\begin{array}{l}\text { Tras la actividad. Darse cuenta de la importancia de realizar una acción } \\
\text { docente dirigida y orientada a fines concretos }\end{array}$ & 4,26 & 0,788 \\
\hline $\begin{array}{l}\text { Tras la actividad. Darse cuenta de la importancia de adaptarse } \\
\text { a las necesidades que surgen en la práctica sin dejarse guiar por la } \\
\text { improvisación }\end{array}$ & 4,11 & 0,907 \\
\hline $\begin{array}{l}\text { Antes de la actividad. Organizarse con el grupo y aportarles } \\
\text { conocimientos y capacidad de trabajo para planificar la actividad }\end{array}$ & 4,1 & 1,141 \\
\hline Antes de la actividad. Preparar materiales específicos para las sesiones & 4 & 1,241 \\
\hline $\begin{array}{l}\text { Antes de la actividad. Usar la información de las carpetas asignadas } \\
\text { a cada niño para obtener información valiosa y tratar de planificar } \\
\text { objetivos de trabajo claros }\end{array}$ & 3,26 & 1,065 \\
\hline
\end{tabular}

Grado de adquisición de competencias relacionadas con la dimensión social

Para este apartado se pregunta a los alumnos si estar con personas de otra cultura les ha ayudado a conocer mejor una realidad diferente, para lo cual el $93.4 \%$ ha señalado que sí, explicando los motivos en caso afirmativo.

En cuanto a la segunda pregunta referida al componente social, el $86.9 \%$ de los alumnos han indicado que la actividad les ha ayudado a conocer mejor las necesidades lingüísticas y sociales de este tipo de alumnado que como futuro profesor necesitarán tener en cuenta, dada la gran diversidad que existe en las aulas.

\section{Asociación}

En cuanto a la relación entre las competencias académicas y las habilidades y actitudes se observa una relación positiva y significativa entre tener en cuenta que las actividades estuvieran orientadas al logro de los objetivos y percibir la influencia que tiene la coordinación y la organización con los compañeros en la práctica educativa $(\mathrm{r}=.497, p<.001)$. Del mismo modo existe relación positiva y significativa entre, al planificar la actividad, tener en cuenta las orientaciones metodológicas estudiadas en la asignatura y los siguientes aspectos: darse cuenta de la importancia de adaptarse a las necesidades que surgen en la práctica $(r=.515, p<.001)$, darse cuenta de la importancia de realizar una acción docente dirigida y orientada a fines $(r=.547$, $p<.001$ ) y darse cuenta de la influencia que tiene la coordinación y organización con mis compañeros en la práctica educativa $(r=.517, p<.001)$. 
Se observa que existe una relación positiva y significativa entre considerar que las orientaciones metodológicas estudiadas ayudan a proceder mejor durante la actividad y darse cuenta de la importancia de realizar una acción docente dirigida y orientada a unos fines $(r=.590, p<.001)$.

\section{Inferenciales}

Comparación de puntuaciones en función del año académico y el curso

Los resultados indican que existen diferencias significativas entre las puntuaciones obtenidas por el alumnado en los siguientes aspectos (ver Tabla 5):

Se encuentran diferencias significativas entre los alumnos del año académico 2013-2014 y 2014-2015 con puntuaciones mayores en estos últimos en preparar materiales específicos para las sesiones $(z=-3.57 ; p<.001)$, organizarse con el grupo y aportarles conocimientos y capacidad de trabajo para planificar la actividad $(z=-3.14 ; p<.005)$, o coordinarse y organizarse con los compañeros en la práctica educativa $(z=-2.86 ; p<.005)$.

En este sentido podríamos decir que los alumnos del curso académico 20142015, parece que aprovecharon mejor la actividad y tuvieron en cuenta estos aspectos. Quizá simplemente debido a una mayor insistencia de las profesoras en estos aspectos fruto de su experiencia vivida el curso anterior.

Tabla 5. Diferencia de medias en función del año académico

\begin{tabular}{lccc}
\hline \multicolumn{1}{c}{ Competencias participativas desarrolladas } & $\begin{array}{c}\text { Año } \\
\text { académico }\end{array}$ & M & DT \\
\hline Preparar materiales específicos para las sesiones & $2013-2014$ & 3.42 & 1.40 \\
\cline { 2 - 4 } & $2014-2015$ & 4.58 & .672 \\
\hline $\begin{array}{l}\text { Organizarse con el grupo y aportarles conocimientos } \\
\text { capacidad de trabajo para planificar la actividad }\end{array}$ & $2013-2014$ & 3.61 & 1.30 \\
\cline { 2 - 4 } & $2014-2015$ & 4.58 & .672 \\
\hline $\begin{array}{l}\text { Coordinarse y organizarse con los compañeros en la } \\
\text { práctica educativa }\end{array}$ & $2013-2014$ & 4.13 & .718 \\
\hline
\end{tabular}

Del mismo modo, se observan diferencias entre el curso académico en el que están los alumnos. De este modo observamos diferencias significativas (Tabla entre los alumnos de $3^{\circ}$ y $5^{\circ}$ curso a favor de estos últimos en que durante la actividad han tenido presente el objetivo que perseguían $(z=-4.20 ; p<.001)$ y las orientaciones metodológicas estudiadas les han ayudado a proceder mejor $(z=-3.16$; $p<.001)$ y que al planificar la actividad, han tenido en cuenta las orientaciones metodológicas estudiadas en la asignatura $(z=-3.55 ; p<.001)$. Por tanto, los alumnos de $5^{\circ}$ curso en relación al componente académico de conocimientos adquiridos puntúan más alto, a diferencia de sus compañeros de $3^{\circ}$, en los enunciados que hacen referencia planificación de la tarea (ver Tabla 6). 
Tabla 6. Diferencia de medias en función del curso académico

\begin{tabular}{lccc}
\hline \multicolumn{1}{c}{ Conocimientos adquiridos } & Curso & M & DT \\
\hline $\begin{array}{l}\text { Durante la actividad. Tener presente el objetivo u } \\
\text { objetivos que perseguían }\end{array}$ & $3^{\circ}$ & 3.44 & .629 \\
\cline { 2 - 4 } $\begin{array}{l}\text { Al planificar la actividad. Tener en cuenta las orien- } \\
\text { taciones metodológicas estudiadas en la asignatura }\end{array}$ & $5^{\circ}$ & 4.67 & .488 \\
\hline $\begin{array}{l}\text { Durante la actividad. las orientaciones metodológi- } \\
\text { cas estudiadas les han ayudado a proceder mejor }\end{array}$ & $5^{\circ}$ & 3.75 & .447 \\
\cline { 2 - 4 } & $5^{\circ}$ & 3.53 & .516 \\
\hline
\end{tabular}

\section{Discusıón}

A lo largo de la asignatura se estudian los contenidos acerca de las habilidades lingüísticas necesarias para el aprendizaje de segundas lenguas ya que su práctica es el principal medio para alcanzar la competencia comunicativa. No obstante lo que determina de una manera muy evidente el aprendizaje en contextos de diversidad lingüística es el tratamiento que el profesor hace de las habilidades lingüísticas a través de la metodología empleada y los contenidos seleccionados para el diseño de las actividades. Precisamente la asignatura profundiza en estos conocimientos referidos al planteamiento de objetivos, diseño de actividades y orientaciones metodológicas para la actividad docente en contextos de diversidad lingüística, que los alumnos han podido aplicar en su trabajo de aprendizaje-servicio.

Como se observa en los resultados (ver Tabla 2) se puntuaron más los elementos cuyo contenido incluye la acción reflexiva posterior a la actividad. Esto es, los alumnos adquieren los conocimientos de la asignatura sobre todo gracias a la reflexión que tiene lugar tras la experiencia y no tanto en su preparación o ejecución. A pesar de que tuvieron la posibilidad de aplicar en una experiencia práctica los contenidos estudiados relacionados con el planteamiento de objetivos, el diseño de actividades y las orientaciones metodológicas para la diversidad lingüística, es tras la experiencia cuando valoran su importancia y toman conciencia de cómo pueden optimizar el proceso de enseñanza-aprendizaje de los niños.

En relación a las competencias participativas desarrolladas, cabe resaltar las tres competencias participativas más valoradas por los alumnos: el trabajo en equipo, la toma de decisiones y la capacidad de reflexión.

Tal como queda reflejado en las respuestas de los alumnos, la toma de decisiones, la asocian sobre todo a la necesidad de adaptación que experimentaron durante la puesta en marcha de las sesiones previamente planificadas. Así muchos tuvieron que "ir adaptando sobre la marcha lo planificado a la realidad que nos encontrábamos en la sesión para que el aprendizaje fuera mejor". Esta competencia participativa les permitió "valorar qué era lo mejor y lo peor en cada caso", ajustando así su acción docente a la realidad del grupo y "ante imprevistos, cambiando lo que teníamos pensado".

Por otro lado, muchos alumnos resaltaron la coordinación entre compañeros como uno de los aprendizajes alcanzados en relación al trabajo en equipo: "tuvi- 
mos que coordinarnos para que la actividad fuera coherente". Sitúan la coordinación como un principio de la profesión docente, "claramente hay que trabajar en equipo para que haya un hilo conductor en las actividades de cada día".

Los alumnos que participaron en la actividad vieron necesario coordinarse desde el planteamiento de la actividad para conducirla con éxito y de manera más específica experimentaron la necesidad de colaboración y de comunicación intragrupal durante la realización de la actividad: "hacemos muchos trabajos en grupo pero en este había que coordinarse delante de los niños incluso, ser paciente y cooperar en todo momento con mis compañeros para que saliera bien". Especialmente ponen de manifiesto el desarrollo de la capacidad de trabajo en equipo posteriormente, por ejemplo, "hablando sobre cómo podía cada uno atender mejor a sus alumnos", "compartiendo experiencias entre todos y dándonos consejos unos a otros", e incluso algunos señalan que hicieron "una crítica constructiva del trabajo que otros compañeros habían realizado".

Por último, la iniciativa y la proactividad la asocian al momento de asumir el control de la actividad trabajando con los niños. Entre los motivos resaltan que la iniciativa es importante "al explicar las actividades y enfrentarse a cómo dirigirlas" y al tratarse de una situación real "no había que esperar a que nos dijeran qué hacer, sino directamente hacerlo".

En relación con el componente social, en primer lugar, existe un gran acuerdo en afirmar que la actividad les ha posibilitado conocer una realidad cultural distinta. Se puede apreciar que la mayoría de las respuestas aportadas por los alumnos aluden a componentes concretos de la competencia comunicativa intercultural (Vila, 2007), como es el conocimiento de similitudes y diferencias de la propia cultura respecto a otras o la empatía y la motivación hacia la convivencia con las personas de origen cultural diferente.

Desconocer las diferencias culturales puede conducir al fracaso en la comunicación intercultural. Ser consciente de la diversidad cultural supone conocer en qué nos diferenciamos y en qué nos parecemos: "he conocido las diferencias respecto a mi cultura a través de sus expresiones y costumbres".

La mayoría de las respuestas se pueden agrupar en la categoría empatía. Esta supone la capacidad de interpretar desde los referentes culturales de otras personas, en este caso de los niños: "he visto cómo ven ellos la realidad que les rodea y cómo lo viven", "he visto otra manera de ver las cosas, otras preocupaciones vitales, otras necesidades", "me he dado cuenta de la realidad desde una perspectiva más cercana y personal".

Tener motivación para comunicarse con personas de referentes culturales distintos supone predisposición e interés en relacionarse. En este sentido bastantes respuestas apuntaban hacia lo que cada uno podía aportar en el encuentro intercultural: "tienes que saber adaptarte también a ellos y conocer su cultura, que es diferente en muchos aspectos".

\section{Conclusiones}

El aprendizaje-servicio como estrategia metodológica adquiere pleno sentido en el marco actual de la educación en competencias y el EEES facilitando la adquisición de distintos tipos de aprendizajes. 
En la experiencia realizada no es tanto el diseño de la actividad ni su ejecución, sino la acción reflexiva posterior lo que ha favorecido la adquisición de los contenidos de la asignatura. El aprendizaje-servicio ha favorecido la disposición a la reflexión en la formación inicial de los docentes y esto es un primer paso para que el futuro profesor adquiera hábitos reflexivos. Las destrezas y las actitudes reflexivas se promueven mediante estrategias metodológicas que ayudan a relacionar la teoría y la práctica. Contar con unos referentes del contexto real de la práctica les ha ayudado a que el contenido adquiera significación y sentido y tomar conciencia sobre la intencionalidad que da sentido a sus acciones.

Por otro lado, cabe resaltar el importante papel en la figura del docente de las competencias participativas:

En primer lugar, los alumnos destacaron la ejercitación en la toma de decisiones durante la actividad. El docente en el aula realiza un proceso de planificación, dirección y control de las actividades de aprendizajes de acuerdo a un diseño curricular. La toma de decisiones cumple un papel fundamental para dar solución a situaciones que se pueden presentar en el aula y orientar el proceso educativo de la forma más eficiente.

En segundo lugar, y de manera específica, los alumnos vivenciaron cómo la coordinación, la colaboración y la comunicación grupal redundan en el éxito personal. Este aprendizaje tiene especial relevancia actualmente, ya que en el marco de las competencias básicas, es muy importante el diálogo en los equipos de docentes para adoptar criterios metodológicos firmes y compartidos que respondan a su vez a los compromisos del Proyecto Curricular.

En tercer lugar, la iniciativa y la proactividad aparecen especialmente valoradas en el control y la dirección de la actividad docente.

Por último, desde una perspectiva social, la actividad de aprendizaje servicio, ha favorecido en los alumnos algunos aspectos de la competencia comunicativa intercultural. Tanto en su vertiente cognitiva, en los aspectos más conceptuales sobre las diferencias culturales y comunicativas, como en su vertiente afectiva, en la empatía y la motivación hacia referentes culturales distintos. Principalmente los alumnos han destacado la parte más emocional y actitudinal.

A pesar de las dificultades que conlleva el planteamiento de esta actividad en su inicio y desarrollo y los obstáculos que en ocasiones hay que ir salvando, a los alumnos les ayuda a conocer mejor las necesidades lingüísticas y sociales de este alumnado que como futuros profesores necesitan tener en cuenta. Aportar sus conocimientos en la atención lingüística de este grupo de niños les ha hecho emplear recursos metodológicos para sacar lo mejor de cada niño. En este sentido han tomado conciencia de la diversidad lingüística como una realidad que se encuentra en las aulas que necesitarán afrontar desde un buen conocimiento de las necesidades lingüísticas en primer lugar y desde una atención específica a cada niño.

\section{RefERENCIAS BIBLIOGRÁFICAS}

Bernstein, B. (1993). La estructura del discurso pedagógico. Clases, códigos y control. Madrid: Morata. 
Batlle, R. (2014). La evolución del aprendizaje-servicio en España. Cuadernos de Pedagogía, 450, 57-59.

Billing, S., y Waterman, A. (2014). Studying Service-learning. Innovation in education research methodology. New York: Lawrence Erlbaum Associates.

Bringle, R., y Hatcher, J. (2009). Innovative practices in service-learning and curricular engagement. New directions for Higher Education, 147, 37-46. doi: 10.1002/ he.356

Campo, L. (2010). El aprendizaje servicio en la Universidad como propuesta pedagógica. En M. Martínez (Ed.), Aprendizaje-servicio y responsabilidad social de las universidades (pp. 81-91). Madrid: Octaedro.

Cerrillo, R., García, R., y López, H. (2013). El aprendizaje servicio como innovación docente en la universidad para la enseñanza de la organización escolar. En M.C. Pérez Fuente y M. M. Molero Jurado (Coords.), Variables psicológicas y educativas para la intervención en el ámbito escolar (pp. 265-270). Almería: Asociación Universitaria de Educación y Psicología.

Cornish, L., y Perkins, K. (2012). Encouraging teacher development through embedding reflective practice in assessment. Asia-Pacific Journal of Teacher Education, 40(2), 159-170. doi: 10.1080/1359866X.2012.669825.

Declaración de Bolonia de 19 de junio de 1999. Recuperado de http://www.educacion.gob.es/boloniaeees/que.html (accedido el 13 de mayo de 2014).

Folgueiras, P., Luna, E., y Puig, G. (2013). Aprendizaje y servicio: estudio del grado de satisfacción de estudiantes universitarios. Revista de Educación, 362, 1-17. doi: 10-4438/1988-592X-RE-2011-362-157.

Furco, A. (1999). Is service-learning really better than community service? A study of high school service program outcome. En A. Furco y S.H. Billing (Eds.). Servicelearning: the essence of pedagogy (pp. 23-50). Greenwich, CT: Information Age.

Gil, J., Chiva, O., y Mark, M. (2014). El aprendizaje de contenidos de educación física en la universidad mediante el aprendizaje-servicio. Tándem, 44, 15-26.

Halstead, A. (1998). Educación redefinida: la promesa del aprendizaje-servicio. En El servicio a la comunidad como aprendizaje escolar (pp. 23-24). Buenos Aires: Ministerio de Educación de la Nación.

Hérbert, A., y Hauf, P. (2015). Student learning through service-learning: effects on academic development, civic responsability, interpersonal skills and practical skills. Active learning in Higher Education, 16(1), 37-49. doi: 10.1177/1469787415573357.

Howard, J.P.F. (2003). Service-learning research: foundational issues. En S.H. Billing y A.S. Waterman (Eds.). Studying Service-learning innovations in Education Research Metholodogy (pp. 1-12). Mahwah, NJ: Lawrence Erlbaum Associates.

LEY ORGÁNICA 4/2007, de 12 de abril, por la que se modifica la Ley Orgánica 6/2001, de 21 de diciembre, de universidades (BOE nº 89, de 13 de abril de 2007).

Lucas, S. (2012). Ética y construcción de toma de decisiones socialmente responsables (identidad personal, social y vocacional): hacia una nueva teoría educativa y de investigación. Méjico: UNAM y Díaz Santos.

Martínez-Odría, A. (2005). Voluntariado: un estudio interdisciplinar. CD-ROM editado por el Instituto de Ciencias de la Educación, Universidad Complutense de Madrid-Universidad y Universidad de Barcelona. 
Martínez-Odría, A. (2006). Service-Learning. Connecting Universities and Communities. Critical Review of the literature. The Spanish case. Proyecto CIVICUS (Leonardo Da Vinci). Proyecto de cooperación universitaria promovido por la Comisión Europea y coordinado por la Vytautas Magnus University (Lituania).

Pérez-Gómez, A. (2010). La naturaleza del conocimiento práctico y sus implicaciones en la formación de docentes. Infancia y Aprendizaje, 33(2), 171 177. doi: 10.1174/021037010791114652. http://www.tandfonline.com/doi/ pdf/10.1174/021037010791114652.

Puig, J.M., Batlle, R.; Bosch, C., y Palos, J. (2007). Aprendizaje Servicio. Educar para la ciudadanía. Barcelona: Octaedro.

Real Decreto 1393/2007, de 29 de octubre, por el que se establece la ordenación de las enseñanzas universitarias oficiales (BOE n² 260, de 30 de Octubre de 2007).

Rodríguez, M. (2014). El aprendizaje-servicio como estrategia metodológica en la Universidad. Revista Complutense de Educación, 25(1), 95-114.

UNESCO, 2009, Comunicado Conferencia Mundial sobre La Educación Superior: La nueva dinámica de la educación superior y la investigación para el cambio social y el desarrollo, París.

Vila, R. (2007). Comunicación intercultural. Madrid: Narcea. 